\title{
An audit on the outcome of children referred to the paediatric cardiology unit with a previous echocardiographic diagnosis of mitral valve prolapse
}

\author{
M P B Goonetilleke ${ }^{1}$, U A Jayarathna ${ }^{2}$, P M W Madanayake ${ }^{2}$, H A M Inthisar ${ }^{2}$, D R Palangasinghe ${ }^{2}$, P S \\ Hettiarachchi $^{2}$, U K Egodage ${ }^{2}$
}

Sri Lanka Journal of Child Health, 2013; 42(3): 144-146

\begin{abstract}
Objective: To determine the outcome of children referred to the paediatric cardiology unit with a previous echocardiographic diagnosis of mitral valve prolapse (MVP).
\end{abstract}

Method: This study was carried out at the paediatric cardiology clinic of Teaching Hospital Karapitiya from $1^{\text {st }}$ January 2012 to $31^{\text {st }}$ December 2012. All children (18 years or less) presenting with a previous echocardiographic diagnosis of MVP were included in study. An echocardiographic diagnosis of MVP was reached based on the standard diagnostic criteria using an IE 33 Phillips echocardiography machine.

Results: There were 141 children presenting with a previous echocardiographic diagnosis of MVP of whom $50.4 \%$ were male. Seventeen percent were below 5 years, $45 \% 5-9$ years, 36\% $10-14$ years and $2 \%$ above 14 years. Of the $141,53.2 \%$ did not have a cardiac murmur, $41.8 \%$ had a grade 2 cardiac murmur and $5 \%$ had a grade 3 murmur. Only 7 (5\%) were confirmed to have MVP using standard diagnostic criteria. Of these 7 cases, 3 did not have a murmur and the remaining 4 had a grade 3 murmur best heard in the mitral area. Out of 141 subjects $57.5 \%$ had undergone 2 previous echocardiographic evaluations and 40.4\% had 3-6 previous echocardiographic evaluations.

Conclusion: This study shows gross over diagnosis of MVP when the standard diagnostic criteria to diagnose MVP are applied.

(Key words: Outcome; children; echocardiographic diagnosis of mitral valve prolapse)

\section{Introduction}

Mitral valve prolapse (MVP) is a valvular heart disease characterized by the displacement of the mitral valve leaflets into the left atrium during

${ }^{1}$ Consultant Paediatric Cardiologist,
${ }^{2}$ Medical
Officer, Paediatric Cardiology Unit, Teaching
Hospital,. Karapitiya

(Received on 15 March 2013: Accepted after revision on 19 April 2013) systole. True prolapse is defined when the whole or part of mitral valve leaflets are displaced more than $2 \mathrm{~mm}$ above the mitral annulus ${ }^{1,2}$.

Mild MVP carries a low risk of complications. In severe cases of MVP, complications include mitral regurgitation, infective endocarditis, congestive heart failure, and in rare circumstances cardiac arrest usually resulting in sudden death. MVP may occur with greater frequency in individuals with Ehlers-Danlos Syndrome, Marfan syndrome or polycystic kidney disease. Other risk factors include Graves disease and chest wall deformities such as pectus excavatum. A correlation has been reported between bipolar disorder and MVP. For unknown reasons, MVP patients tend to have a low body mass index (BMI) and are typically leaner than individuals without $\mathrm{MVP}^{3,4}$.

Upon auscultation of an individual with MVP, a mid-systolic click followed by a late systolic murmur, heard best at the apex, is common. In contrast to most other heart murmurs, the murmur of MVP is accentuated by standing and Valsalva manoeuvre (earlier systolic click and longer murmur) and diminished with squatting (later systolic click and shorter murmur). The only other heart murmur that follows this pattern is the murmur of hypertrophic cardiomyopathy ${ }^{1,5}$.

Prior to the strict criteria for the diagnosis of MVP, the incidence of MVP in the general population varied greatly. Some studies estimated the incidence of MVP at 5 to 15 percent or even higher $^{6,7}$. Recent elucidation of mitral valve anatomy and the development of three-dimensional echocardiography have resulted in improved diagnostic criteria, and the true prevalence of MVP based on these criteria is estimated at $2-3 \%^{2,6}$. As part of the Framingham Heart Study, for example, the prevalence of MVP in Framingham was estimated at $2.4 \%{ }^{2,6}$.

Echocardiography is the most useful method of diagnosing a prolapsed mitral valve. Two and 3 dimensional (2D \& 3D) echocardiography are particularly valuable, as they allow visualization of the mitral leaflets relative to the mitral annulus. This allows measurement of the leaflet thickness and their displacement relative to the annulus. Thickening of the mitral leaflets $>5 \mathrm{~mm}$ and leaflet 
displacement $>2 \mathrm{~mm}$ above the mitral annulus indicates classic mitral valve prolapse $e^{7,8}$.

Generally, MVP is benign. However, MVP patients with a murmur, not just an isolated click, have an increased mortality rate of $15-20 \%{ }^{1,6}$. The major predictors of mortality are the severity of mitral regurgitation and the ejection fraction ${ }^{1,6}$.

Following the establishment of the Paediatric Cardiology Unit at Teaching Hospital (TH) Karapitiya we started receiving a substantial number of children and adolescents with a previous echocardiographic diagnosis of MVP for evaluation. Most of these children were healthy and asymptomatic individuals with adequate growth for age. When echocardiography was performed strictly using the standard diagnostic criteria, most of these children did not have confirmed MVP. Therefore our team decided to carry out this study to demonstrate the actual outcome of children who were referred for evaluation with a previous echocardiographic diagnosis of MVP.

\section{Method}

This study was carried out at the Paediatric Cardiology Clinic of $\mathrm{TH}$ Karapitiya from $1^{\text {st }}$ January 2012 to $31^{\text {st }}$ December 2012. This is the only tertiary care cardiac referral centre for the southern province with a monthly patient turnover of around 300. Therefore the patient population is scattered throughout the southern province of Sri Lanka. All children (including adolescents) presenting with a previous echocardiographic diagnosis of MVP were included in this study. The children who were diagnosed for the first time as MVP by our team were excluded from the study.

Patient details such as age, sex, weight and number of previous echocardiograms performed in the past were recorded in a data sheet by a medical officer. A full cardiovascular system examination was performed by the Consultant Paediatric Cardiologist and it was recorded whether a murmur was present or absent. If a murmur was present the grade of the murmur and the area it was best heard was recorded. A complete 2D echocardiography was performed using a Phillips IE33 echocardiography machine by the Consultant Paediatric Cardiologist obtaining the standard views and modified views when needed. Special attention was paid to parasternal long axis views and apical four chamber views where mitral valve assessment could be performed optimally. After the echocardiographic evaluation it was recorded whether there was a diagnosis of MVP in line with the standard diagnostic criteria (whole or part of either anterior mitral leaflet or posterior mitral leaflet or both leaflets move above the mitral annulus during ventricular systole) or was a normal echocardiographic evaluation if this criterion was not fulfilled. After data collection, data were entered into an access database, exported to SPSS and analysed using SPSS statistical analysis software.

Informed and written consent was taken from the parents or the guardian of children who were included in this study. All records were kept with the principal investigator and no personal information was divulged to a third party. There were no potential hazards as maximum confidentiality was maintained during the study. Patients were not subjected to any invasive procedures.

\section{Results}

There were 141 children (18 years or less) presenting with a previous echocardiographic diagnosis of MVP. Of this 70 (49.7\%) were female and $71(50.4 \%)$ were male. Twenty three $(17 \%)$ patients were less than 5 years of age, 65 (45\%) 5-9 years, $50(36 \%) 10-14$ years and $3(2 \%)$ over 14 years of age.

In this cohort we noted that $75(53.2 \%)$ did not have a cardiac murmur on clinical examination while $59(41.8 \%)$ had a grade 2 cardiac murmur and $7(5 \%)$ had a grade 3 murmur. None had grades $1,4,5$ or 6 murmurs.

When standard diagnostic criteria were applied, only $7(5 \%)$ of the cases were confirmed to have MVP, 134 (95\%) not fulfilling the diagnostic criteria. Of the 7 cases with a confirmed diagnosis of MVP, 3 did not have a murmur and 4 had a grade 3 murmur best heard in the mitral area.

Out of the 141 subjects $81(57.5 \%)$ had undergone two previous echocardiographic evaluations, 43 $(30.5 \%)$ had 3 previous echocardiographic evaluations, $13(9.2 \%)$ had 4 previous echocardiographic evaluations and $1(0.7 \%)$ had 6 previous echocardiographic evaluations.

\section{Discussion}

MVP is clearly defined in standard cardiology textbooks as prolapse of either or both mitral valve leaflets $2 \mathrm{~mm}$ above the mitral annulus. This conclusion should be drawn by looking at the mitral valves in apical four chamber view and parasternal long axis view in 2D echocardiography since apical four chamber view per se will give a false negative impression of MVP. In addition MVP is a progressive disease which does not get naturally corrected during the course of life. 
We strictly applied these standard diagnostic criteria throughout our study and all echocardiograms were performed by a single paediatric cardiologist with substantial echocardiography experience to avoid individual bias. All individuals included in the study had a previous echocardiographic diagnosis of MVP. The echocardiographer was not aware of the person or the level of skill the previous echocardiographer had to diagnose MVP and this strategy was adopted to avoid further bias of the echocardiographer who could thus reach an independent and unbiased diagnosis of MVP. Using these strategies we found that $134(95 \%)$ of the 141 cases assessed did not have MVP which amounts to $95 \%$ being falsely diagnosed as MVP previously. To support this fact our data shows $53.2 \%$ of cases did not have any murmur which negates the diagnosis of a MVP clinically in $53.2 \%$ of the studied population.

Although only $7(5 \%)$ confirmed cases of MVP were found in this study $81(57.5 \%)$ had undergone two previous echocardiographic evaluations and 57 (40.4\%) had 3-6 previous echocardiographic evaluations. This implies a knowledge gap in terms of correctly diagnosing MVP.

During our study we observed that most children and their families who had a previous false positive diagnosis of MVP encountered many psychosocial issues. Most parents were overprotective of their children and had not allowed them to participate in normal physical activities both at home and in the school.

Therefore this study has revealed a gross over diagnosis of MVP by means of echocardiography in terms of standard laid down criteria to diagnose MVP. This may be attributed to lack of awareness of the standard laid down criteria to diagnose MVP.

\section{Conclusions and recommendations}

- This study shows a gross over diagnosis of MVP within this study population when the standard diagnostic criteria to diagnose MVP are applied.

- A thorough awareness and capacity building programme will be needed to make echocardiographers aware of the standard diagnostic criteria of MVP.

\section{References}

1. Anders S, Said S, Schulz F, Puschel K. Mitral valve prolapse syndrome as cause of sudden death in young adults. Forensic Science International 2007; 171:127-30. http://dx.doi.org/10.1016/j.forsciint.2006.10.011
2. Avierinos JF, Gersh BJ, Melton LJ, Bailey KR, Shub C, Nishimura RA, et al. Natural history of asymptomatic mitral valve prolapse in the community. Circulation 2002; 106:1355-61. http://dx.doi.org/10.1161/01.CIR.0000028933. $\underline{34260.09}$

3. Enriquez-Sarano M, Akins CW, Vahanian A. Mitral regurgitation. Lancet 2009; 373:138294.

http://dx.doi.org/10.1016/S01406736(09)6069 $\underline{2-9}$

4. Gelb BD. Marfan's syndrome and related disorders--more tightly connected than we thought. New England Journal of Medicine 2006; 355(8):841-4. http://dx.doi.org/10.1056/NEJMe068122

5. Carabello BA. The current therapy for mitral regurgitation. Journal of the American College of Cardiology 2008; 52:319-26.

http://dx.doi.org/10.1016/j.jacc.2008.02.084

6. Zuppiroli A, Rinaldi M, Kramer-Fox R, Favili $\mathrm{S}$, Roman MJ, Devereux RB. Natural history of mitral valve prolapse. American Journal of Cardiology 1995; 75:1028-32. http://dx.doi.org/10.1016/S00029149(99)8071 $\underline{8-8}$

7. Freed LA, Levy D, Levine RA, Larson MG, Evans JC, Fuller DL, et al. Prevalence and clinical outcome of mitral-valve prolapse. New England Journal of Medicine 1999; 341:1-7. http://dx.doi.org/10.1056/NEJM199907013410 $\underline{101}$

8. Lambert AS, Miller JP, Merrick SH, Schiller NB, Foster E, Muhiudeen-Russell I, Cahalan MK. Improved evaluation of the location and mechanism of mitral valve regurgitation with a systematic trans-esophageal echocardiography examination. Anesthesia Analgesia 1999; 88 (6):1205-12. http://dx.doi.org/10.1097/00000539199906000 $\underline{-00004}$ 